\title{
FOXD1 Promotes Cell Growth and Metastasis by Activation of Vimentin in NSCLC
}

\author{
Dan Li $^{\mathrm{a}}$ Suyun Fan ${ }^{\mathrm{a}} \quad$ Fei Yu ${ }^{\mathrm{a}} \quad \mathrm{Xuchao}_{\mathrm{Zhu}}^{\mathrm{a}}$ Yingchun Song ${ }^{\mathrm{a}}$ \\ Meng Yea Lihong Fan ${ }^{\mathrm{b}}$ Zhongwei Lva \\ aDepartment of Nuclear Medicine, Shanghai Tenth People's Hospital, Tongji University School of \\ Medicine, Shanghai, 'Department of Respiration, Shanghai Tenth People's Hospital, Tongji University \\ School of Medicine, Shanghai, China
}

\section{Key Words}

NSCLC $・$ FOXD1 $・$ Vimentin $•$ Cell migration $•$ Cell invasion

\begin{abstract}
:
Background/Aims: Forkhead box D1 (FOXD1) has a well-established role in early embryonic development and organogenesis and functions as an oncogene in several cancers. However, the clinical significance and biological roles of FOXD1 in non-small cell lung cancer (NSCLC) remain largely unknown. Methods: A total of 264 primary NSCLC tissue samples were collected. The expression levels of FOXD1 in these samples were examined by immunohistochemical staining. The expression of FOXD1 was knocked down by lentiviral shRNA. The relative expression of FOXD1 was determined by qRT-PCR, Western blotting and immunofluorescence image. The functional roles of FOXD1 in NSCLC were demonstrated cell viability CCK-8 assay, colony formation, cell invasion and migration assays, and cell apoptosis assay in vitro. In vivo mouse xenograft and metastasis models were used to assess tumorigenicity and metastatic ability. The Chi-square test was used to assess the correlation between FOXD1 expression and the clinicopathological characteristics. Survival curves were estimated by Kaplan-Meier method and compared using the log-rank test. The Cox proportional hazards model was used for univariate and multivariate analyses. Results: We determined that higher levels of FOXD1 were present in NSCLC tissues, especially in metastatic NSCLC tissues. FOXD1 was also higher in all NSCLC cells compared with normal human bronchial epithelial cells. A higher expression level of FOXD1 was associated with malignant behavior and poor prognosis in NSCLC patients. Knockdown of FOXD1 significantly inhibited proliferation, migration, and invasion in vitro and tumor growth and metastasis in vivo, and it increased the apoptosis rates of NSCLC cells. Mechanistic analyses revealed that FOXD1 expressed its oncogenic characteristics through activating Vimentin in NSCLC. Multivariate Cox regression analysis indicated that FOXD1 was an independent prognostic factor both for overall survival (OS) and disease-free survival (DFS)

D. Li, S. Fan and F. Yu contributed equally to this work.

\begin{tabular}{ll}
\hline Lihong Fan & Department of Nuclear Medicine, Shanghai Tenth People's Hospital \\
and Zhongwei Lv & Shanghai (China) \\
& E-Mail fanlih@aliyun.com; Izwkxy@163.com
\end{tabular}
\end{abstract}


in NSCLC patients. Conclusion: Our results indicated that FOXD1 might be involved in the development and progression of NSCLC as an oncogene, and thereby might be a potential therapeutic target for NSCLC patients.

(c) 2018 The Author(s)

Published by S. Karger AG, Basel

\section{Introduction}

Lung cancer is the leading cause of cancer deaths worldwide [1] and has become the second leading cause of death in China, with an increased incidence of $465 \%$ over the past 30 years [1,2]. Non-small cell lung cancer (NSCLC) accounts for over $80 \%$ of all lung cancer cases, and approximately $70 \%$ of NSCLC patients are diagnosed with advanced disease [1, 3]. Although surgical resection and adjuvant therapy have improved in recent years, the 5-year survival rate of NSCLC remains poor due to the lack of obvious symptoms for early diagnosis, recurrence and metastasis [3, 4]. Hence, a better understanding of the molecular mechanisms of NSCLC development and progression will facilitate the development of new targets for therapy.

FOXD1, a member of the forkhead family, is primarily expressed in human embryonic tissues, where it regulates organogenesis [5]. Furthermore, FOXD1 was found to be a mediator and indicator of cell reprogramming by mediating self-renewal and differentiation $[6,7]$. Increasing evidence has indicated that cell reprogramming is associated with tumor initiation and progress [8]. For example, FOXD1 was found to be increased in prostate cancer tissues and associated with lymph node metastasis [9]. Another study has shown that FOXD1 promoted cell proliferation and chemotherapy resistance by inducing the G1 to $S$ phase transition in breast cancer [10]. FOXD1 was reported to be significantly upregulated in glioma samples, and further investigations suggested that FOXD1 directly regulated ALDH1A3 transcription to activate clonogenic and tumorigenic potential of mesenchymal stem-like cells of glioma [8]. These findings strongly indicated that FOXD1 enrichment in tumor cells might affect biological functions, including cell proliferation, apoptosis, invasion, and metastasis. Interestingly, FOXD1 was also reported to be overexpressed in human NSCLC, and patients with high FOXD1 expression survived for a significantly shorter time than those with low FOXD1 expression [11]. However, the exact roles and molecular mechanisms of FOXD1 contributing to NSCLC progress have remained largely unknown.

In our present study, we first confirmed that the expression of FOXD1 was significantly increased in NSCLC tissues. Both in vitro and in vivo assays showed that short hairpin RNA (shRNA)-mediated FOXD1 knockdown inhibited NSCLC cell proliferation, invasion, and metastasis. Mechanistic studies showed that FOXD1 promoted NSCLC cell invasive potential by upregulating the expression of Vimentin. Our results provide an important mechanism of FOXD1 regulation of cell proliferation and invasion during NSCLC progression.

\section{Materials and Methods}

\section{Patients and samples}

A total of 264 primary NSCLC cases who underwent surgical resection of NSCLC confirmed by pathological evaluation were collected at the Department of Respiration of Shanghai 10th People's Hospital affiliated with Tongji University in Shanghai, China. Paired NSCLC tissue and adjacent normal lung tissue samples were obtained from these 264 patients between Jan 2007 and Dec 2010. None of the patients had received adjuvant chemotherapy, radiation or any other treatment before resection, and all patients were followed-up. All tumor tissues were classified according to the 2010 lung cancer staging system of the AJCC [12]. Written informed consent was obtained from all patients. The use of these tissue specimens was approved by the ethics committee of Shanghai 10th People's Hospital and conducted in adherence with the Declaration of Helsinki protocols. Specimens were frozen in liquid nitrogen and stored at $-80{ }^{\circ} \mathrm{C}$ before use. 


\section{Cellular Physiology Cell Physiol Biochem 2018:51:2716-2731

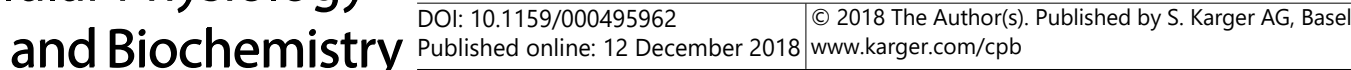

Li et al.: FOXD1 Mediates Vimentin in NSCLC

Immunohistochemical analysis

Immunohistochemical (IHC) staining was performed using the Dako Envision system (Dako, Carpinteria, CA, USA) according to the manufacturer's instructions. In short, paraffin-embedded tissues were cut into 3-5 $\mu \mathrm{m}$ sections for dewaxing and rehydration. The sections were microwaved in $0.1 \mathrm{M}$ citrate buffer $(\mathrm{pH}=6.0)$ and incubated in $3 \% \mathrm{H}_{2} \mathrm{O}_{2}$ at room temperature for $30 \mathrm{~min}$ followed by incubation with $10 \%$ goat serum at room temperature for $30 \mathrm{~min}$. Primary antibody (anti-FOXD1 rabbit polyclonal antibody, 1:40, Proteintech, Chicago, IL, USA; mouse anti-vimentin, 1:400, Zymed Laboratories, USA) was added and the sections were incubated overnight at $4{ }^{\circ} \mathrm{C}$. Then, biotin-labeled secondary antibody (CW Bio, Beijing, China) was added and the sections were incubated at room temperature for $30 \mathrm{~min}$. Next, the sections were immersed in freshly prepared 3, 3'-diaminobenzidine (DAB) for color development for 5 min. The sections were then thoroughly rinsed with water and stained with hematoxylin. Five high-power fields $(400 \times)$ were randomly selected for each specimen slide. When the cellular membrane and/or cytoplasm were stained brown, the cells were positive.

\section{Cell culture}

Five NSCLC cell lines, A549, 95-C, 95-D, NCI-H460, and NCI-H1299, and a normal human bronchial epithelial cell line (16HBE) were obtained from the Institute of Biochemistry and Cell Biology of the Chinese Academy of Sciences (Shanghai, China). The cells were cultured in DMEM supplemented with $10 \%$ fetal bovine serum (FBS) (Invitrogen, Carlsbad, USA), $100 \mu \mathrm{g} / \mathrm{ml}$ streptomycin and $100 \mathrm{units} / \mathrm{ml}$ penicillin (HyClone, Logan, USA) in a humidified atmosphere with $5 \% \mathrm{CO}_{2}$ at $37^{\circ} \mathrm{C}$.

\section{Cell transfection and lentivirus production}

The lentiviral shRNA constructs for FOXD1 knockdown were purchased from Sigma-Aldrich (St. Louis, MO, USA), the sequences of the FOXD1 shRNA was 5 ' - C C GG T T G T TAATA AC G C TAT G T TAGC T C G AG C TAAC ATAG C G T TAT TAA CAAT T T T T G-3', while the sequences of specificity control for the shRNA was 5'-CCGGAATGCCTACGTTAAGCTATACCTCGAGGTATAGCTTAACGTAGGCATTTTTTTG-3'. The lentivirus of shFOXD1 was generated by co-transfecting HEK293T with the pPACKH1 Lentivector Packaging KIT (SigmaAldrich). To generate the stable cell line, cells were transfected with lentiviruses for $24 \mathrm{~h}$ and replaced with complete culture medium. The Vimentin-siRNA (siVimentin) and negative control siRNA were all purchased from Santa-Cruz Biotechnology (Dallas, TX, USA). Approximately $1 \times 10^{5}$ cells were plated in 6-well dishes before transfection and grown without antibiotics. Cells were transfected using Lipofectamine ${ }^{\circledR}$ RNAiMAX reagent (Invitrogen) according to the protocol. Cells were analyzed by Western blot analysis 24-48 h after transfection.

\section{RNA extraction and quantitative real-time PCR}

Total RNA was extracted from cells using TRIzol reagent (Invitrogen) according to the manufacturer's protocol. Reverse transcription was performed with the Superscript first-strand synthesis system (Invitrogen, Carlsbad, USA) according to the manufacturer's instructions. The following primers used for real-time PCR were as follows: FOXD1 forward, 5'-AAGAACCCGCTGGTGAAG-3' and reverse, 5'-GTCCAGTAGTTGCCCTTGC-3'; and GAPDH forward, 5'-ACAACTTTGGTATCGTGGAAGG-3' and reverse, 5'GCC ATCACGCCACAGTTTC-3'. The relative expression of FOXD1 mRNA was normalized to the expression level of GAPDH mRNA. qRT-PCR was performed on an ABI 7900HT instrument (Applied Biosystems, USA). All of the determinations were performed in triplicate and all experiments were carried out at least three independent times.

\section{Cell viability, colony formation and apoptosis assays}

Cell viability was measured using the Cell Counting Kit 8 (CCK8, Dojindo, Japan) according to the manufacturer's instructions. In brief, cells were seeded in 96-well plates at a density of $1 \times 10^{3}$ cells/well in $100 \mu \mathrm{l}$ of medium. Then, $100 \mu \mathrm{l}$ of CCK-8 was added to the culture at 1, 2, 3 and 4 days. The optical density (OD) was measured at $450 \mathrm{~nm}$ in a spectrophotometer (Spectra Max Plus, Molecular Devices, Sunnyvale, USA). 


\section{Cellular Physiology Cell Physiol Biochem 2018;51:2716-2731 \begin{tabular}{l|l|l|l} 
and BOI: 2018 The Author(s). Published by S. Karger AG, Basel \\
and
\end{tabular}}

Li et al.: FOXD1 Mediates Vimentin in NSCLC

Cells were collected in the logarithmic phase of growth and seeded into 6-well plates at a density of 800 cells/ well for 14 days. The medium was refreshed with medium containing $10 \%$ FBS once every 3 days. The cells were then fixed with $4 \%$ paraformaldehyde for $30 \mathrm{~min}$ and stained with crystal violet (Shanghai Jingtian Biotech Co., Ltd., JT-00518) for 20 min. Representative photographs were captured, and colonies with more than 40 cells were counted.

Apoptotic cells were examined using an Annexin V-PE Apoptosis Detection Kit (Millipore, USA). The samples were harvested and then stained with $5 \mu \mathrm{l}$ of Annexin V-PE and $5 \mu \mathrm{l}$ of PI in the dark for 15 min. Cellular apoptosis was detected by flow cytometry and the results were analyzed using the Cell-QuestTM Pro software (BD Biosciences, MA, USA).

\section{Migration and invasion assays}

Cells $\left(3 \times 10^{5} /\right.$ well) were suspended in $100 \mu \mathrm{l}$ of serum-free DMEM and added to the upper chamber of an insert ( $8 \mu \mathrm{m}$ pore size, Millipore, USA) coated with or without Matrigel (BD) for the transwell migration and invasion assay. Then, $600 \mu \mathrm{l}$ DMEM with $10 \%$ FBS was added to the lower chamber. Cells were fixed and stained with $0.05 \%$ crystal violet after $36 \mathrm{~h}$ of incubation at $37^{\circ} \mathrm{C}$ for the migration and invasion assays. The stained cells were counted in 6 random fields of each chamber under a microscope $(\times 100$ magnification $)$ and photographed using an IX71 inverted microscope (Olympus, Tokyo, Japan) at 200× magnification.

\section{Immunofluorescence}

For immunofluorescence microscopy, the cells treated with shFOXD1 or SCR were cultured on cover slips and fixed with 4\% (vol/vol) paraformaldehyde (Sigma-Aldrich) for $15 \mathrm{~min}$ and then permeabilized in 0.1\% Triton X-100 (Sigma-Aldrich). The cells were washed with a 2\% glycine solution and blocked with PBS containing $2 \% \mathrm{FBS}$ and $0.5 \%$ saponin for $30 \mathrm{~min}$ at $37^{\circ} \mathrm{C}$ The primary antibody against vimentin $(1: 100$, CST, Danvers, MA, USA) was added and incubated overnight at $4{ }^{\circ} \mathrm{C}$, followed by incubation with Alexa Flour 488-conjugated secondary antibodies (1:1000, Sigma, St. Louis, MO, USA) for 1 h. DAPI (Sigma, St. Louis, MO, USA) was used to counterstain DNA. Immunofluorescence images for Vimentin were viewed with confocal laser-scanning microscopy (Fluoview FV1000, Olympus, Japan).

\section{Western blot analysis}

The cultured cells were harvested and lysed with RIPA lysis buffer (Promega) for $30 \mathrm{~min}$ at $4{ }^{\circ} \mathrm{C}$. A total of $60 \mu \mathrm{g}$ of total protein was loaded into 10\% SDS-PAGE and transferred electrophoretically onto a PVDF membrane (Millipore, Germany). The membranes were blocked with $1 \%$ bovine serum albumin (BSA) in TBST buffer (Tris Buffer Saline containing 0.1\% Tween-20) for $1 \mathrm{~h}$ at room temperature and subsequently incubated with primary antibodies against FOXD1 (Proteintech) or E-cadherin, N-cadherin, Vimentin (Santa Cruz Biotechnology Inc. Dallas, TX, USA) or MMP-9, MMP-2, GAPDH (Cell Signaling Technology, USA) overnight at $4^{\circ} \mathrm{C}$. After extensive washing with TBST buffer, the blots were then probed with horseradish peroxidase-conjugated secondary antibody for $1 \mathrm{~h}$ at room temperature. Protein bands were detected by the enhanced chemiluminescence reagent ECL (Millipore, MA, USA) and the intensity of the bands was analyzed using ImageJ software (National Institutes of Health, USA).

\section{In vivo tumor formation and metastasis assays in nude mice xenograft model}

Six-week-old male nude BALB/c mice (4-6 weeks old; body weight, 18-22 g) were purchased from the Shanghai Laboratory Animal Center of the Chinese Academy of Sciences (Shanghai, China). Nude mice were injected subcutaneously into the left flanks with NCI-H460 cells $\left(1 \times 10^{7}\right)$ infected with lentiviral constructs carrying either the shFOXD1 vector or the control vector. Two perpendicular diameters (a: the largest; b: the smallest) of the tumor were measured with a caliper for 5 weeks monitoring. Tumor volume (V) was calculated using the following formula: $\mathrm{V}=\mathrm{a} \times \mathrm{b}^{2} \times 0.5$. Tumor weights were also measured. All the mice were euthanized 5 weeks later. Mouse xenograft tumor tissues were harvested and tissue sections were then subjected to IHC staining with antibodies against FOXD1 and ki-67 (Sigma-Aldrich). Mouse lungs were fixed with $10 \%$ neutral-buffered formalin, embedded in paraffin, sectioned at $4 \mu \mathrm{m}$ and stained with H\&E. The number of pulmonary metastatic tumor nodules was counted under a light microscope at magnifications of $\times 100$ and $\times 200$.

\section{KARGER}




\section{Cellular Physiology Cell Physiol Biochem 2018;51:2716-2731

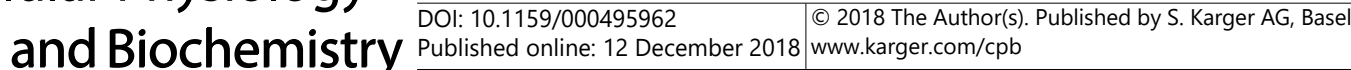

Li et al.: FOXD1 Mediates Vimentin in NSCLC

Statistical analysis

The data were assessed using SPSS 18.0 software (IBM, SPSS, Chicago, IL, USA). All of the experiments were performed in triplicate, and Student's t-test was used to analyze the significance of the different levels between two groups. The Chi-square test was used to assess the correlation between FOXD1 expression and the clinicopathological characteristics. Survival curves were estimated by the Kaplan-Meier method and compared using the log-rank test. The Cox proportional hazards model was used for univariate and multivariate analyses. The data are expressed as the mean \pm standard deviation (SD) and differences of $P<0.05$ were considered to be statistically significant.

\section{Results}

FOXD1 is frequently upregulated in human NSCLC

The mRNA expression level of FOXD1 was determined in 264 pairs of NSCLC tissue and paired adjacent non-tumor tissue by qRT-PCR. As shown in Fig. 1A, FOXD1 was significantly increased in NSCLC tumor tissue compared with the paired non-tumor tissue. Furthermore, the mRNA level of FOXD1 was significantly higher in NSCLC patients with metastasis than in patients without metastasis (Fig. 1B). FOXD1 protein expression was confirmed by IHC staining. Our results showed that non-tumor lung tissues were stained slightly positive for FOXD1, with positive staining of FOXD1 in NSCLC tissue, especially in NSCLC tissues with metastasis (Fig. 1C). Finally, protein expression levels of FOXD1 were measured in five NSCLC cell lines and 16HBE cell by Western blotting. Expression levels of FOXD1 were elevated in all NSCLC cell lines compared to 16HBE cells, especially in NCI-H460 and NCI-H1299 cells, which have the highest endogenous FOXD1 levels (Fig. 1D). These results suggested that FOXD1 might act as an oncogene in the development of NSCLC.

\section{Knockdown of FOXD1 inhibits the proliferation of NSCLC}

Since FOXD1 was upregulated in NSCLC tissues, lentiviral-mediated FOXD1 knockdown was used to assess the effect of FOXD1 on cell proliferation. First, shRNA\#1, \#2, and \#3 stable silencing FOXD1 were established in NCI-H460 and NCI-H1299 cell lines. Western blotting showed that FOXD1 was obviously knocked down in both shRNA\#1 cell lines (Fig. 2A). Then, shRNA\#1 (named shFOXD1) was used in the following experiments. Cell viability was significantly reduced in both stable FOXD1-knockdown NCI-H460 and NCI-H1299 cell lines compared with SCR by the CCK-8 assay (Fig. 2B). Consistent with the results of CCK8 , the results of the colony formation assays indicated that the stable FOXD1-knockdown NSCLC cells exhibited a weakened capacity for colony formation compared with SCR (Fig. 2C). Next, flow cytometric analysis was performed to further evaluate the effects of FOXD1 on the proliferation of NSCLC cells by altering cell apoptosis. Silencing FOXD1 significantly increased the percentage of apoptosis rates compared with SCR (Fig. 2D). Collectively, our data from the FOXD1-knockdown cells indicated that FOXD1 promoted NSCLC cell proliferation by affecting cell apoptosis.

\section{FOXD1 silencing impairs NSCLC cell migration and invasion}

Uncontrolled cell proliferation and metastasis are the main characteristics of tumor aggressive behaviors [13-15]. It is necessary to evaluate the effects of FOXD1 on the migratory and invasive capacity of NSCLC cells. Transwell migration and Matrigel invasion assays showed that deletion of FOXD1 significantly decreased the number of migrated and invaded NCI-H460 and NCI-H1299 cells (Fig. 3A). Since our data suggest the potential involvement of FOXD1 in the metastatic capacity of the cancer phenotype, we explored whether FOXD1 regulates gene expression of markers of metastasis. Interestingly, FOXD1 silencing resulted in an obvious decrease in the protein expression of Vimentin, N-cadherin, MMP-2 and MMP9, while endogenous FOXD1 knockdown caused an increase in E-cadherin. These data might explain the underlying mechanisms of FOXD1-induced cell invasion. 


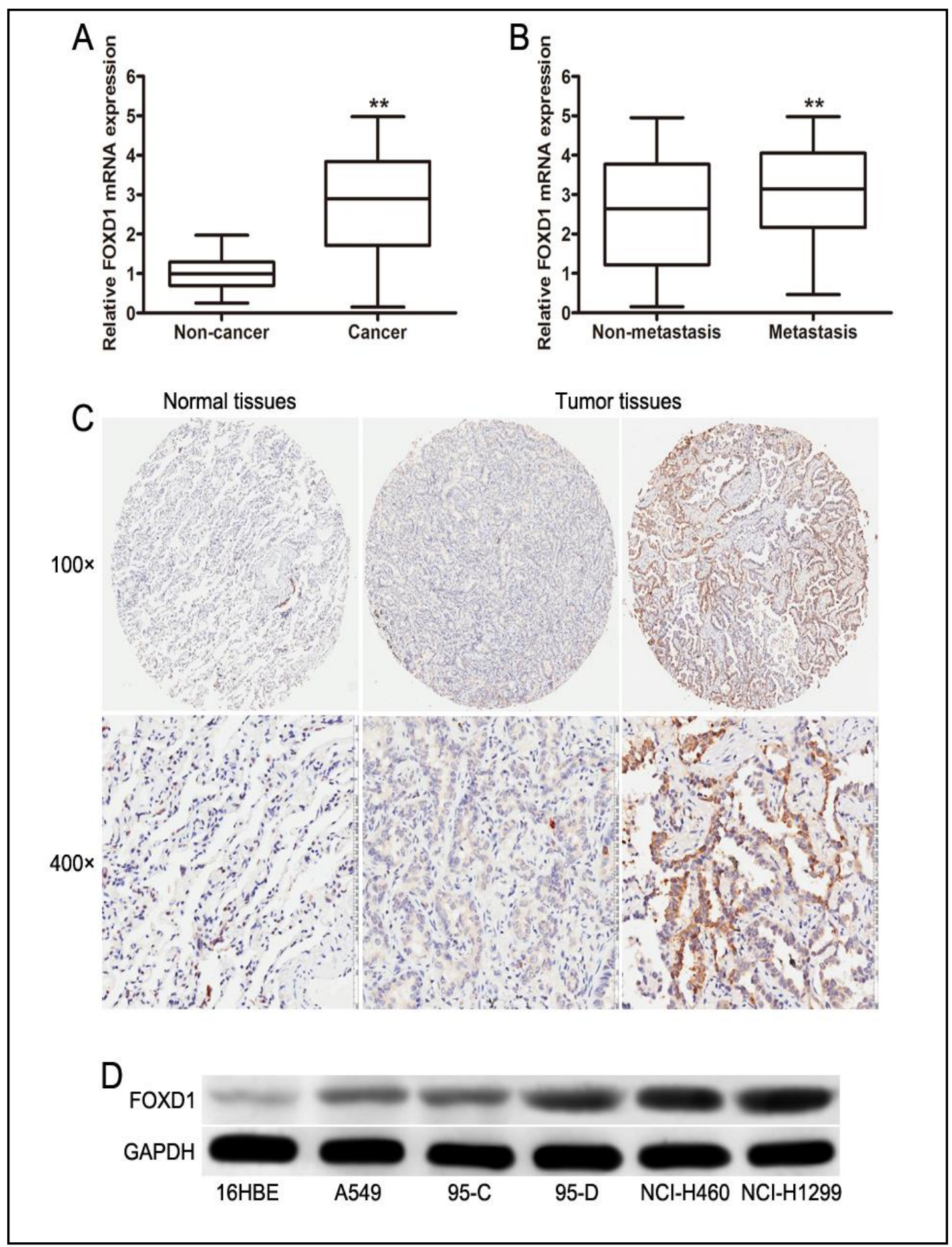

Fig. 1. Expression of FOXD1 is elevated in NSCLC tissues and cells. (A) qRT-PCR was performed to detect the FOXD1 mRNA expression in 264 pairs of NSCLC tissues and corresponding noncancerous lung tissues. (B) The mRNA level of FOXD1 was measured in primary NSCLC cancer tissues of 115 patients without metastasis and 149 patients with metastasis. (C) FOXD1 protein expression in normal lung tissues and NSCLC was detected by immunohistochemistry. Left, normal lung tissue; middle, weakly positive staining of FOXD1 in NSCLC tissue; right, strongly positive staining of FOXD1 in NSCLC tissue. (D) Western blots of FOXD1 expression in the 16HBE cell line and the A549, 95-C, 95-D, NCI-H460, and NCI-H1299 NSCLC cell lines. ${ }^{* *} \mathrm{P}<0.01$.

\section{KARGER}




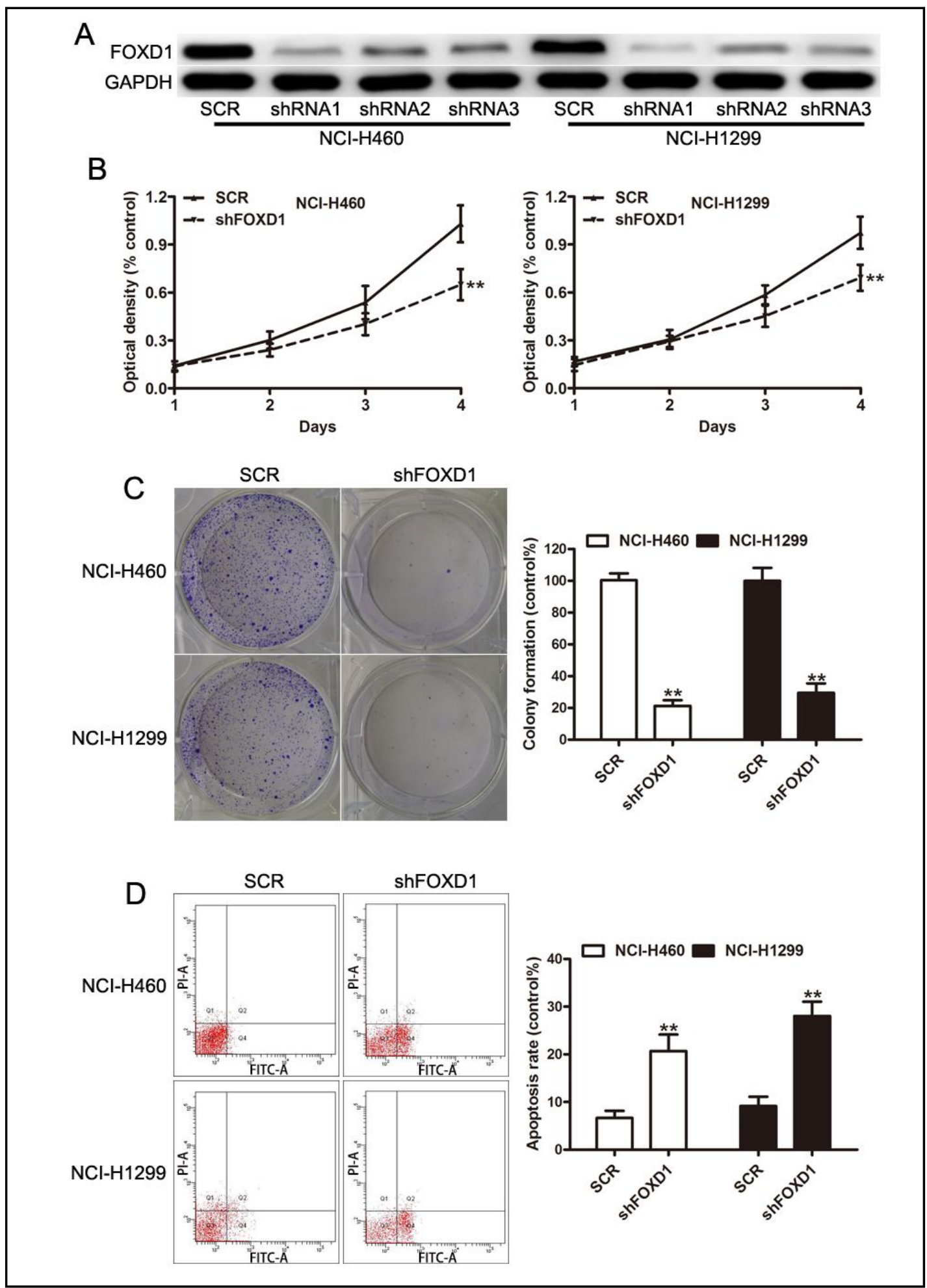

Fig. 2. Down-regulation of FOXD1 inhibits NSCLC proliferation. (A) The expression of FOXD1 in NCI-H460 and NCI-H1299 cells is interfered by lentiviral-mediated FOXD1 shRNA. Western blotting was performed to confirm the knockdown efficiency. GAPDH was used as the loading control for Western blotting. The (B) CCK-8 and (C) the clone formation assay detected cell proliferation after transducing with FOXD1 shRNA. (D) Cell apoptosis analysis revealed that FOXD1 has influenced the apoptosis of NCI-H460 and NCI-H1299 cells. ${ }^{* *} \mathrm{P}<0.01$.

\section{KARGER}




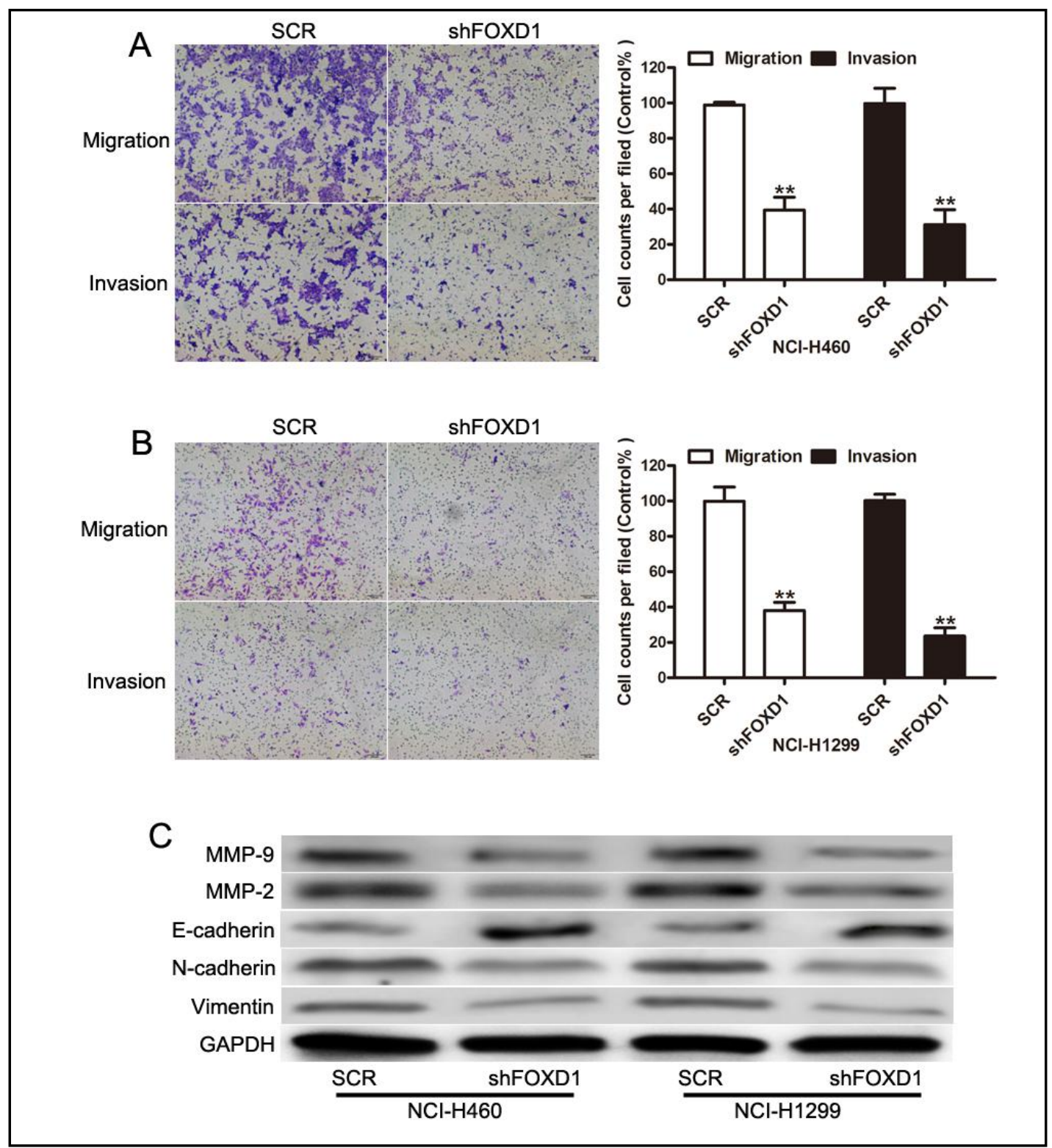

Fig. 3. FOXD1 knockdown decreases NSCLC cell migration and invasion. Representative images and quantification of migration and invasion of NCI-H460 (A) and NCI-H1299 (B) cells after transducing with FOXD1 shRNA. (C) The protein expression of Vimentin, N-cadherin, E-cadherin, MMP-2 and MMP-9 in NCI-H460 and NCI-H1299 cells transduced with FOXD1 shRNA or SCR. ${ }^{* *} \mathrm{P}<0.01$.

FOXD1 depletion in NSCLC cells results in impaired tumor growth and metastasis in vivo

Given that NSCLC cells infected with ShFOXD1 exhibited reduced proliferative capacity, migratory and invasive capacity in vitro, we further confirmed the effects of FOXD1 on the proliferative and metastatic capacity of NSCLC cells in vivo. NCI-H460 cells infected with shFOXD1 or SCR were injected subcutaneously into the left flanks of nude mice to produce a xenograft model. Gross specimens dissected from the mice pictorially demonstrate this difference in volume (Fig. 4A). Both tumor volume and weight were measured to analyze the tumor growth. The nude mice implanted with NCI-H460 cells treated with lentiviral shFOXD1 vector showed significantly smaller $(P<0.01)$ (Fig. 4B) and lighter $(P<0.01)$ (Fig. 4C) implantation tumors than nude mice that had been implanted with NCI-H46 cells treated with the control vector. Ki-67 is an excellent marker to determine the growth of a tumor cell, and

\section{KARGER}




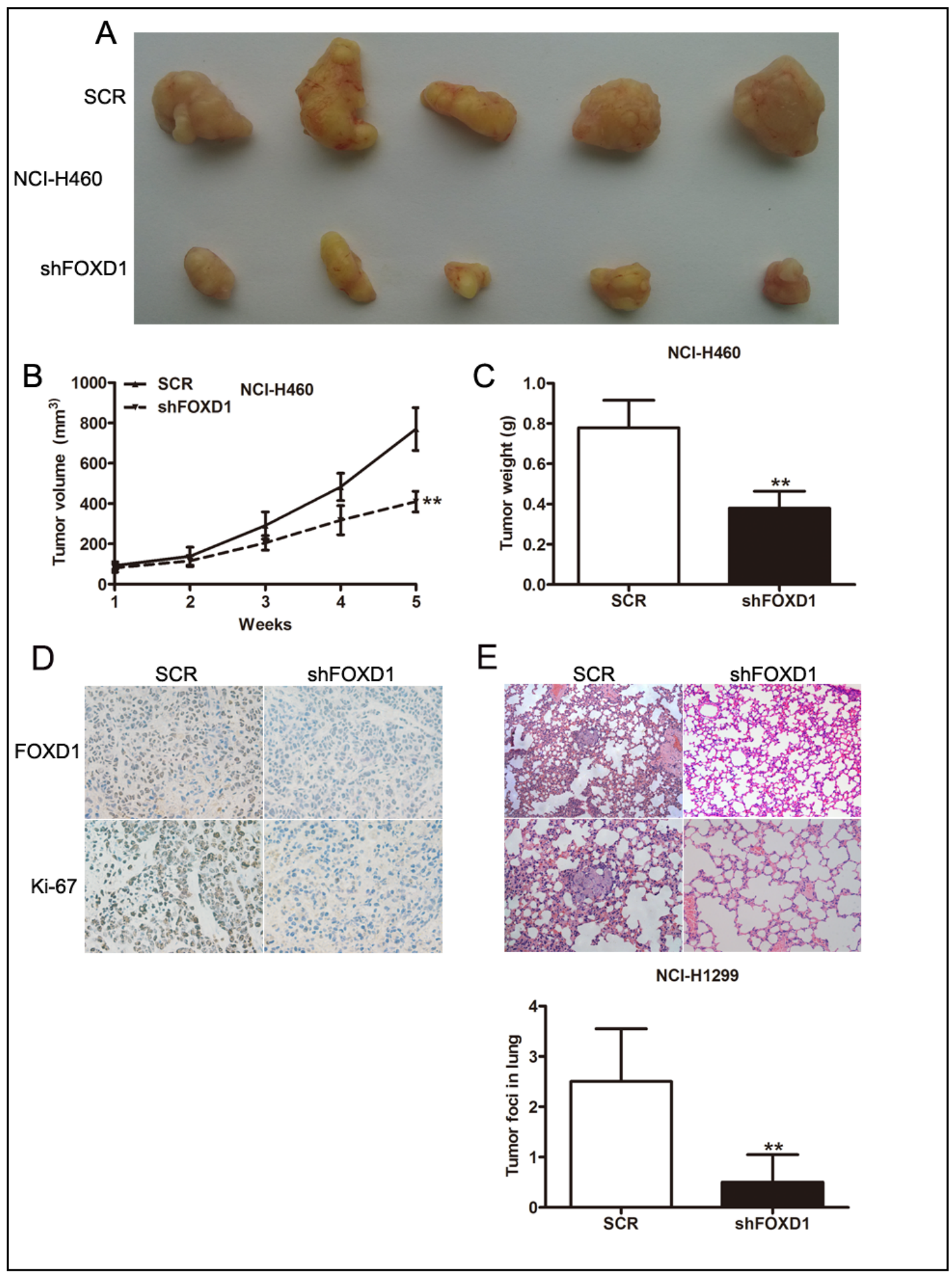

Fig. 4. Knockdown of FOXD1 represses tumor growth and metastasis in vivo. (A) Representative images of tumors formed by NCI-H460 isolated from nude mice. (B) Tumor cell growth course in nude mice. (C) Tumor weight in nude mice. (D) Representative images of FOXD1 and Ki-67 staining in tumors isolated from nude mice. (E) Representative H\&E stained images of metastatic nodes from mouse lungs. ${ }^{* *} \mathrm{P}<0.01$. 


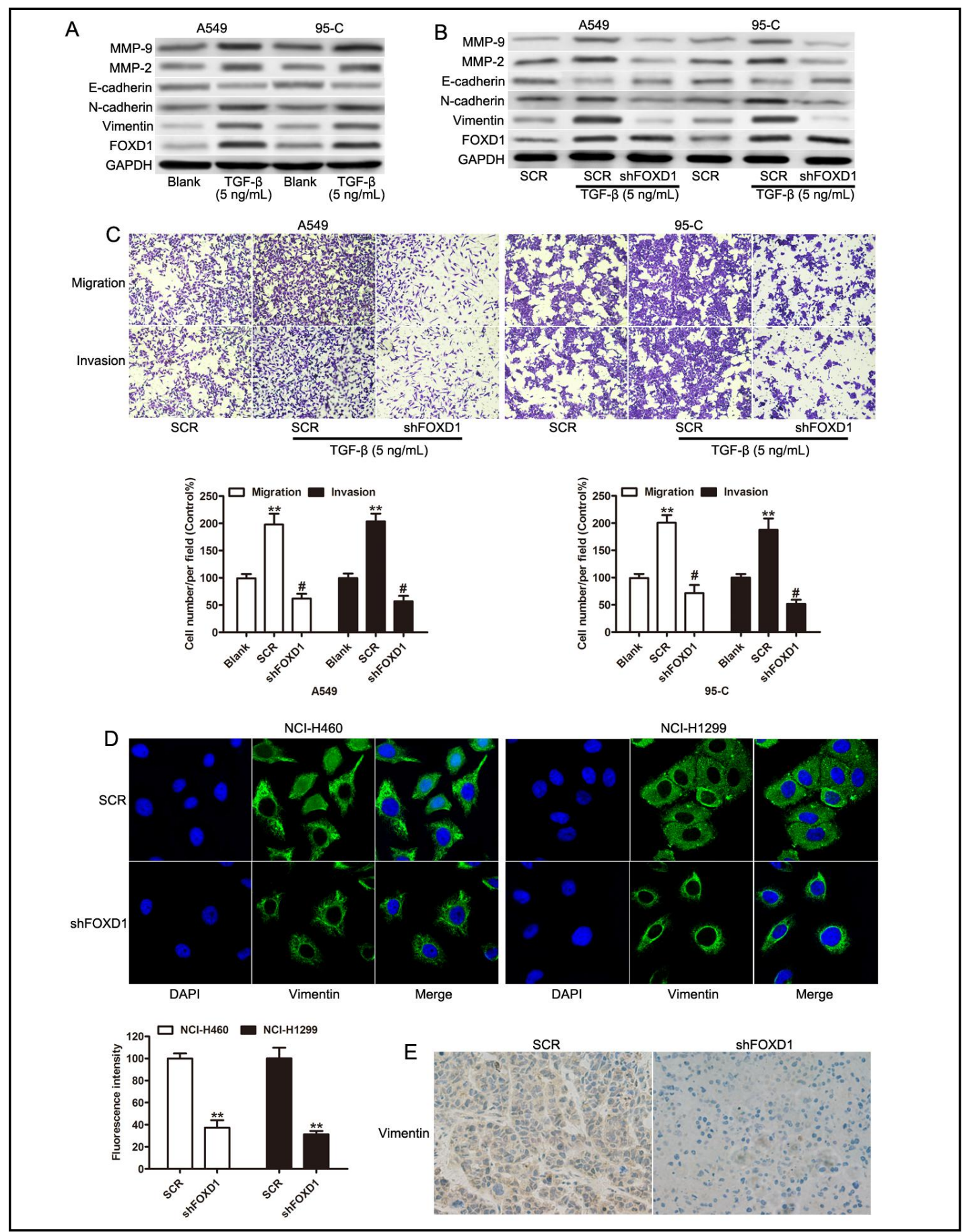

Fig. 5. FOXD1 promotes NSCLC cell migration and invasion through Vimentin. (A) N-cadherin, E-cadherin, MMP-2, MMP-9, FOXD1 and Vimentin expression was increased during TGF- $\beta 1$ (48 h) induced cell migration and invasion in A549 and 95-C cells. (B) The protein expression of FOXD1, Vimentin, N-cadherin, E-cadherin, MMP-2 and MMP-9 in A549 and 95-C cells transfected with negative control (NC) or Vimentin siRNA after TGF- $\beta 1$ stimulation. (C) Representative images and quantification of migration and invasion of A549 and 95-C cells transfected with negative control (NC) or Vimentin siRNA after TGF- $\beta 1$ stimulation. (D) Immunofluorescence analysis of Vimentin in NCI-H460 and NCI-H1299 cells transduced with FOXD1 shRNA or SCR. Green signals indicate that Vimentin and nuclei were counterstained with DAPI. (E) IHC staining showed the expression of Vimentin in tumor xenografts isolated from nude mice. ${ }^{* *} \mathrm{P}<0.01$.

\section{KARGER}


our results show that knockdown of FOXD1 expression caused a significant decrease in the number of Ki-67 positive cells in FOXD1 silencing xenograft tumors compared with SCR (Fig. 4D). Histologic sections of the lungs were obtained from mice that had been implanted with tumor cells and H\&E stained. The number of metastatic nodules was counted under a microscope. The nude mice implanted with NCI-H460 cells infected with shFOXD1 had a significantly lower number of pulmonary metastases than nude mice that had been implanted with NCI-H46 cells treated with the control vector $(P<0.01)$ (Fig. $4 \mathrm{E}$ ). Overall, these results suggested that FOXD1 depletion can inhibit tumor aggressiveness by reducing cell proliferation and metastasis both in vitro and in vivo.

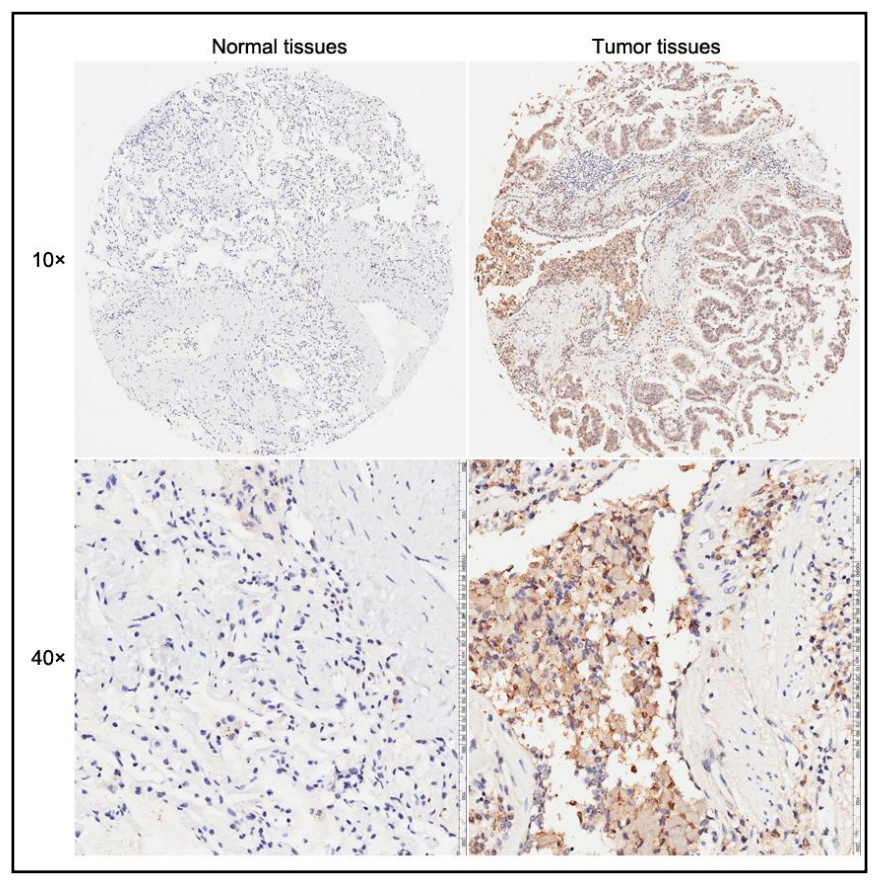

Fig. 6. Representative photomicrographs of the immunohistochemical expression of Vimentin in adjacent normal tissues and NSCLC tumors.

\section{FOXD1 is essential for TGF- $\beta$-induced Vimentin expression, cell migration and invasion}

Previous studies reported that TGF- $\beta$ functions as a major inducer of cancer progression $[16,17]$. Interestingly, a significant increase in FOXD1 in A549 and 95-C cells was observed after treatment with $5 \mathrm{ng} / \mathrm{ml}$ TGF- $\beta$ (Fig. $5 \mathrm{~A}$ ). Vimentin is a widely considered as a marker of the epithelial mesenchymal transition (EMT), which can be induced by TGF- $\beta$ in several cancers $[18,19]$. Consisted with these results, Vimentin was also increased after TGF- $\beta$ treatment, and TGF- $\beta$ also induced higher expression of Vimentin, N-cadherin, MMP-2 and MMP-9 and lower expression of E-cadherin (Fig. 5A). Next, we examined whether FOXD1 regulates the expression of the mesenchymal gene vimentin upon TGF- $\beta$ stimulation. As we expected, FOXD1 silencing decreases TGF- $\beta$-induced expression of Vimentin, N-cadherin, MMP-2 and MMP-9, while increasing the expression of E-cadherin (Fig. 5B). In addition, an in vitro transwell assay showed that knockdown of FOXD1 decreased NSCLC cell migration and invasion induced by TGF- $\beta$ (Fig. 5 C), thus indicating that FOXD1 is involved in TGF- $\beta$ induced migration and invasion. Moreover, knockdown of FOXD1 decreased the intensity (green) of Vimentin in NCI-H460 and NCI-H1299 cells (Fig. 5D). Immunohistochemical analysis of tumor sections indicates low Vimentin expression from FOXD1 silenced tumors compared with SCR tumors (Fig. 5E). These data suggest that FOXD1 promotes NSCLC progress associated with induced vimentin and increased invasive ability.

Combination of FOXD1 and Vimentin exhibits improved prognostic accuracy for NSCLC

We analyzed the relationship between FOXD1 and Vimentin expression and patient survival. The immunohistochemical expression of Vimentin in NSCLC tumors was more positive than the adjacent normal tissues (Fig. 6). Samples were classified into two groups according to the mean value of FOXD1 expression. As shown in Table 1, high FOXD1 expression was significantly associated with the tumor-node-metastasis (TNM) stage of tumor progression. Our results also showed that higher protein levels of Vimentin were significantly associated with tumor histology grade, TNM stage and tumor progression (Table 1). The prognostic value of FOXD1 and Vimentin was analyzed by comparing the OS and DFS 
Table 1. Associations between FOXD1/Vimentin expression level and clinicopathologic features of NSCLC patients $(n=264)$

\begin{tabular}{|c|c|c|c|c|c|c|}
\hline \multirow{2}{*}{ Variables } & \multicolumn{2}{|c|}{$\begin{array}{c}\text { FOXD1 } \\
\text { Expression, } \mathrm{n}\end{array}$} & \multirow[t]{2}{*}{$\mathrm{P}$} & \multicolumn{2}{|c|}{$\begin{array}{c}\text { Vimentin } \\
\text { Expression, } \mathrm{n}\end{array}$} & \multirow[t]{2}{*}{$P$} \\
\hline & $\begin{array}{c}\text { Low } \\
\mathrm{n}=132\end{array}$ & $\begin{array}{c}\text { High } \\
\mathrm{n}=132\end{array}$ & & $\begin{array}{c}\text { Low } \\
n=132\end{array}$ & $\begin{array}{c}\text { High } \\
\mathrm{n}=132\end{array}$ & \\
\hline & & Sex & & & & \\
\hline Female & 13 & 15 & 0.689 & 10 & 18 & 0.110 \\
\hline Male & 119 & $\begin{array}{l}117 \\
\text { Age,y }\end{array}$ & & 122 & 114 & \\
\hline$\leq 60$ & 66 & 68 & 0.806 & 72 & 62 & 0.218 \\
\hline$>60$ & 66 & $\begin{array}{c}64 \\
\text { Histolog }\end{array}$ & & 60 & 70 & \\
\hline Squamous cell carcinoma & 100 & 90 & 0.357 & 96 & 94 & 0.921 \\
\hline Adenocarcinoma & 19 & 27 & & 23 & 23 & \\
\hline Others & 13 & $\begin{array}{c}15 \\
\text { Smoking }\end{array}$ & & 13 & 15 & \\
\hline Yes & 106 & 113 & 0.252 & 108 & 100 & 0.228 \\
\hline \multirow[t]{2}{*}{ No } & \multirow{2}{*}{\multicolumn{3}{|c|}{ Histology grade }} & 24 & 32 & \\
\hline & & & & & & \\
\hline Well & 26 & 24 & 0.338 & 31 & 19 & 0.046 \\
\hline Moderate & 97 & 92 & & 93 & 96 & \\
\hline \multirow[t]{2}{*}{ Poor and Unknown } & 9 & 16 & & 8 & 17 & \\
\hline & & TNM stag & & & & \\
\hline I- II & 67 & 48 & 0.018 & 69 & 46 & 0.004 \\
\hline IIIIV & 65 & 84 & & 63 & 86 & \\
\hline \multicolumn{7}{|c|}{ Tumor progression } \\
\hline Metastasis & 85 & 64 & 0.009 & 93 & 56 & $<0.001$ \\
\hline No metastasis & 47 & 68 & & 39 & 76 & \\
\hline
\end{tabular}

Table 2. Univariate Analyses of Factors Associated with Overall Survival and Disease Free Survival. Abbreviation: 95\% CI, 95\% confidence interval; AFP, alpha-fetoprotein; NS, not significant (Cox proportional hazards regression model)

\begin{tabular}{|c|c|c|c|c|}
\hline \multirow{2}{*}{$\begin{array}{l}\text { Parameter } \\
\text { Variable }\end{array}$} & \multicolumn{2}{|c|}{ Overall Survival } & \multicolumn{2}{|c|}{ Disease Free Survival } \\
\hline & Hazard ratio $(95 \% \mathrm{Cl})$ & $\mathrm{P}$ Value & $\begin{array}{l}\text { Hazard ratio } \\
(95 \% \mathrm{CI})\end{array}$ & $\begin{array}{c}\mathrm{P} \\
\text { Value }\end{array}$ \\
\hline Sex (male vs. female) & $0.883(0.568-1.375)$ & NS & $\begin{array}{l}0.910(0.591- \\
1.402)\end{array}$ & NS \\
\hline Age, $y(>60$ vs. $\leq 60$ ) & $1.099(0.837-1.441)$ & NS & $\begin{array}{l}1.100(0.845- \\
1.433)\end{array}$ & NS \\
\hline Smoking ( yes vs. no) & $1.146(0.819-1.603)$ & NS & $\begin{array}{c}1.149(0.830- \\
1.591)\end{array}$ & NS \\
\hline $\begin{array}{l}\text { Histology (Adenocarcimoma vs Squamous } \\
\text { cell carcinoma) }\end{array}$ & $1.089(0.890-1.333)$ & NS & $\begin{array}{l}1.024(0.833- \\
1.259)\end{array}$ & NS \\
\hline Histology grade (Poor vs. well) & $1.386(1.054-1.823)$ & 0.020 & $\begin{array}{c}1.380(1.053- \\
1.809)\end{array}$ & 0.020 \\
\hline TNM stage ( III/IV vs. I/II) & $1.838(1.387-2.435)$ & $<0.001$ & $\begin{array}{l}1.921(1.461- \\
2.524)\end{array}$ & $<0.001$ \\
\hline $\begin{array}{l}\text { Tumor progression (Metastasis vs. No } \\
\text { metastasis) }\end{array}$ & $1.879(1.428-2.471)$ & $<0.001$ & $\begin{array}{l}2.028(1.552- \\
2.650)\end{array}$ & $<0.001$ \\
\hline FOXD1 ( high vs. low) & $2.044(1.550-2.696)$ & $<0.001$ & $\begin{array}{l}1.954(1.495- \\
2.554)\end{array}$ & $<0.001$ \\
\hline Vimentin (high vs. low) & $2.276(1.720-3.013)$ & $<0.001$ & $\begin{array}{c}2.530(1.922- \\
3.331)\end{array}$ & $<0.001$ \\
\hline
\end{tabular}

Table 3. Multivariate Analyses of Factors Associated with Overall Survival and Disease Free Survival. Abbreviation: 95\% CI, 95\% confidence interval; NS, not significant (Cox proportional hazards regression model)

\begin{tabular}{|c|c|c|c|c|}
\hline \multirow[b]{2}{*}{ Variable } & \multicolumn{2}{|c|}{ Overall Survival } & \multicolumn{2}{|c|}{ Disease Free Survival } \\
\hline & $\begin{array}{l}\text { Hazard ratio }(95 \% \\
\text { CI) }\end{array}$ & P Value & $\begin{array}{l}\text { Hazard ratio }(95 \% \\
\text { CI) }\end{array}$ & P Value \\
\hline Histology grade (Poor vs. well) & $\begin{array}{c}0.989(0.723- \\
1.352)\end{array}$ & NS & $\begin{array}{c}0.899(0.661- \\
1.222)\end{array}$ & NS \\
\hline TNM stage ( III/IV vs. I/II) & $\begin{array}{l}1.426(0.919- \\
2.211)\end{array}$ & NS & $\begin{array}{l}1.383(0.908- \\
2.106)\end{array}$ & NS \\
\hline $\begin{array}{l}\text { Tumor progression (Metastasis vs. No } \\
\text { metastasis) }\end{array}$ & $\begin{array}{l}1.087(0.689- \\
1.715)\end{array}$ & NS & $\begin{array}{l}1.225(0.789- \\
1.901)\end{array}$ & NS \\
\hline FOXD1 ( high vs. low) & $\begin{array}{l}2.017(1.525- \\
2.668)\end{array}$ & $<0.001$ & $\begin{array}{l}1.947(1.484- \\
2.556)\end{array}$ & $<0.001$ \\
\hline Vimentin ( high vs. low) & $\begin{array}{l}1.920(1.306- \\
2.822)\end{array}$ & $<0.001$ & $\begin{array}{l}2.338(1.750- \\
\quad 3.124)\end{array}$ & $<0.001$ \\
\hline \multicolumn{5}{|l|}{ Combination of FOXD1 and Vimentin } \\
\hline Overall & & $<0.001$ & & $<0.001$ \\
\hline $\begin{array}{l}\text { High FOXD1/High Vimentin vs. low } \\
\text { FOXD1/low Vimentin }\end{array}$ & $\begin{array}{l}4.547(2.912- \\
7.102) \\
\end{array}$ & $<0.001$ & $\begin{array}{c}4.696(3.049- \\
7.234) \\
\end{array}$ & $<0.001$ \\
\hline
\end{tabular}

of patients with high and low FOXD1 or Vimentin expression (n=132 each group). KaplanMeier analysis showed that patients with low FOXD1 expression had a significantly higher postoperative 5-year OS and a significantly higher postoperative DFS than patients with high FOXD1 expression (Fig. 7A and 7B) $(P<0.01)$. When patients were divided according to the level of Vimentin expression, those with low Vimentin expression had a significantly higher 5 -year OS and significantly higher 5-year DFS than those with high Vimentin expression (Fig. 7C and 7D) $(P<0.01)$. Univariate analysis showed that FOXD1, Vimentin, histology grade, TNM stage and tumor progression were significantly associated with OS and DFS in NSCLC patients (Table 2). However, sex, age, smoking, and histology did not significantly associate with OS and DFS (Table 2). In addition, multivariate analysis showed that FOXD1 and Vimentin were independent prognostic indicators for both OS and DFS (Table 3). In the combined analysis of FOXD1 and Vimentin expression, patients with low FOXD1 and low Vimentin expression had the highest 5-year OS and the lowest DFS, and the opposite pattern was observed in patients with high FOXD1 and Vimentin expression (Fig. 7E and 7F). Taken 


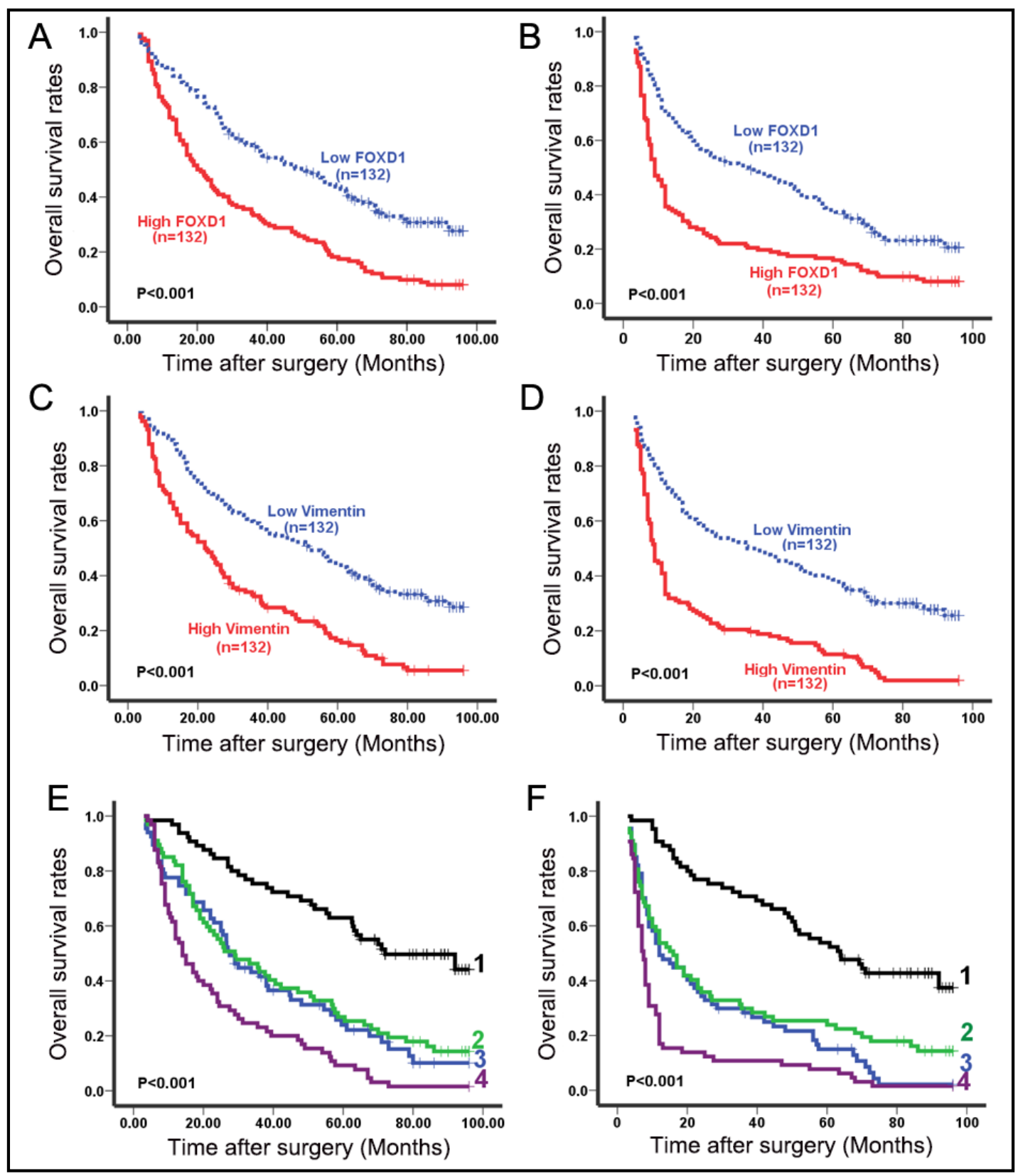

Fig. 7. Prognostic significance of FOXD1 and Vimentin expression in NSCLC patients. (A and B) Kaplan-Meier analysis of the correlation between FOXD1 expression and overall survival (A) and disease-free survival (B) in NSCLC patients. (C and D) Kaplan-Meier analysis of the correlation between Vimentin expression and OS (C) and DFS (D) in NSCLC patients. (E and F) Patients with low FOXD1 and low Vimentin had the longest OS (E) and lowest possibility of DFS (F) according to the cut-off values of FOXD1 and Vimentin, which were defined as the median of the cohort. 1: low FOXD1 and low Vimentin; 2: high FOXD1 and low Vimentin; 3: low FOXD1 and high Vimentin; 4: high FOXD1 and high Vimentin.

together, these results indicate that FOXD1 and Vimentin expression levels are positively correlated with poor OS and a greater recurrence rate in NSCLC patients, suggesting that FOXD1 and Vimentin are prognostic indicators in NSCLC. 


\section{Cellular Physiology Cell Physiol Biochem 2018;51:2716-2731 \\ and Biochemistry \begin{tabular}{l|l} 
DOI: 10.1159/000495962 & (c) 2018 The Author(s). Published by S. Karger AG, Basel \\
www.karger.com/cpb
\end{tabular}}

Li et al.: FOXD1 Mediates Vimentin in NSCLC

\section{Discussion}

The FOX family of genes, characterized by a common 100 amino acid winged-helix DNAbinding domain, regulates a wide spectrum of biological processes, including cell growth, survival, longevity, immunity, metabolism, differentiation, development and migration [20]. Thus, the dysregulation of FOX genes is also observed in a variety of cancerspage 5 . The FOXD1 gene is located on chromosome $5 q 12$ and is involved in a wide array of biological processes, including proliferation, invasion and tumorigenesis $[5,7,8]$. A previous study used microarray datasets from 90 lung cancer specimens to analyze the levels of FOXD1 mRNA and found that the aberrant FOXD1 mRNA was correlated with NSCLC patient survival. They also reported that FOXD1-knockdown led to suppression of cell proliferation and thought that the expression status of FOXD1 is a novel prognostic factor of NSCLC [11].

In this study, we have demonstrated that levels of FOXD1 were increased in clinical NSCLC tissues compared to adjacent normal lung tissues, especially in metastatic NSCLC tissues. FOXD1 was also increased in NSCLC cell lines, including A549, 95-C, 95-D, NCI-H460, and NCI-H1299, compared with normal human bronchial epithelial cells. These results are consistent with a previous study that showed that FOXD1 expression was elevated in NSCLC tissues and NSCLC cell lines, including H358, H520, and H522 [11]. Furthermore, we revealed that a higher expression level of FOXD1 was associated with malignant behavior and poorer prognosis of NSCLC patients. It is worth mentioning that FOXD1 is after all a transcription factor so nuclear localization would be expected, but FOXD1 was mainly in cytoplasm, which was previously reported [8]. However, we don't found that high levels of FOXD1 expression were significantly associated with these clinicopathological findings, including the presence of squamous cell carcinoma, male gender and history of heavy smoking.

To determine the biology function of FOXD1 in NSCLC, gain-and loss-of-function were used. Knockdown of FOXD1 significantly inhibited proliferation, migration, and invasion in vitro and tumorgrowth and metastasis in vivo, and increased the apoptosis rates of NSCLC cells. The results were consistent with other studies, which showed significant growth inhibition in several cancer cell types $[7,10,21]$. In breast cancer, it was suggested that FOXD1 induces the G1/S transition and promotes the cell cycle progression by down-regulation of $\mathrm{p} 27$ expression [10]. In glioma, reduced expression of FOXD1 was associated with the inhibition of glioma cell proliferation, cell survival and migration, implying that FOXD1 is an oncogene in glioma [21]. It was found that knockout of FOXD1 inhibits downstream transcriptional Dax1, which is a component of the autoregulatory network for maintaining pluripotency, and the fate mapping analyses further reveal that $>95 \%$ of induced pluripotent stem cell (iPSC) colonies are derived from FOXD1-positive cells. Thus, FOXD1 is a mediator and indicator of successful progression of reprogramming [7]. It was found that the gene encoding the small leucine-rich proteoglycan decorin is repressed by FOXD1 in cortical interstitial cells, partially rescuing the failure of progenitor cell differentiation [5].

Previous studies have shown that overexpression of vimentin may predict the progression and unfavorable survival of NSCLC [22-24]. Vimentin is essential to the progression and prognosis of cancer through the EMT, a critical event in the invasion, progression and metastasis of epithelial cancers $[25,26]$. In the present study, we found that FOXD1 expression was related to increased expression of Vimentin. Interestingly, high levels of FOXD1 expression were significantly associated with the high level of Vimentin. When the FOXD1 was knocked out, Vimentin expression was down-regulated. The mechanistic analyses revealed that FOXD1 performed its oncogenic characteristics through activating Vimentin in NSCLC.

A meta-analysis of observational studies with 4118 NSCLC cases predicted the prognostic values of Vimentin expression in NSCLC and revealed that Vimentin is a helpful potential biomarker in NSCLC [27], but there is no information regarding the relationship between FOXD1 and Vimentin expression in lung cancer. FOXD1 expression was identified as a prognostic factor in the previous univariate analysis and multivariate Cox analysis [11]. In the present study, the prognosis of patients was also investigated. Patients with high FOXD1 
expression survived for a significantly shorter time than those with low FOXD1 expression. Multivariate Cox regression analysis indicated that FOXD1 was an independent prognostic factor for OS and DFS in NSCLC patients. In addition, overexpression of vimentin may also predict the progression and an unfavorable survival of NSCLC. In the combined analysis of FOXD1 and Vimentin expression, high FOXD1 and Vimentin expression were associated with the worst prognosis. To the best of our knowledge, this is the first study to investigate the relationship between FOXD1 and Vimentin in the prognosis of NSCLC patients.

\section{Conclusion}

In summary, we showed that knockdown of FOXD1 could inhibit cell proliferation and invasion in vitro and in vivo and that high expression of FOXD1 is associated with worse prognosis of NSCLC. Our findings suggest that FOXD1 acts as an oncogenic factor in NSCLC partly by activating Vimentin. Hence, as an independent prognostic factor, FOXD1 could be an innovative treatment target for NSCLC.

\section{Abbreviations}

FOXD1 (Forkhead box D1); NSCLC (non-small cell lung cancer); OS (overall survival); DFS (disease-free survival); ShRNA (short hairpin RNA); IHC (Immunohistochemical); DAB (diaminobenzidine); FBS (fetal bovine serum); CCK8 (Cell Counting Kit 8); OD (optical density); SD (standard deviation); EMT (epithelial mesenchymal transition); TNM (tumornode-metastasis); iPSC (induced pluripotent stem cell.).

\section{Acknowledgements}

This work was funded by the National Science Foundation of China (NSFC), No. 81473469 and No. 81501505.

\section{Disclosure Statement}

The authors declare that they have no competing interests.

\section{References}

1 Jemal A, Bray F, Center MM, Ferlay J, Ward E, Forman D: Global cancer statistics. CA Cancer J Clin 2011;61:69-90.

$>2$ Wen C, Dehnel T: China wrestles with lung cancer. Lancet Oncol 2011;12:15.

-3 Heist RS, Engelman JA: SnapShot: non-small cell lung cancer. Cancer Cell 2012;21:448.e442.

$>4$ Warth A: [Diagnosis, prognosis, and prediction of non-small cell lung cancer. Importance of morphology, immunohistochemistry and molecular pathology]. Pathologe 2015;36 Suppl 2:194-200.

-5 Levinson RS, Batourina E, Choi C, Vorontchikhina M, Kitajewski J, Mendelsohn CL: Foxd1-dependent signals control cellularity in the renal capsule, a structure required for normal renal development. Development 2005;132:529-539.

-6 Fetting JL, Guay JA, Karolak MJ, Iozzo RV, Adams DC, Maridas DE, Brown AC, Oxburgh L: FOXD1 promotes nephron progenitor differentiation by repressing decorin in the embryonic kidney. Development 2014;141:17-27.

7 Koga M, Matsuda M, Kawamura T, Sogo T, Shigeno A, Nishida E, Ebisuya M: Foxd1 is a mediator and indicator of the cell reprogramming process. Nat Commun 2014;5:3197. 


\section{Cellular Physiology Cell Physiol Biochem 2018;51:2716-2731 and Biochemistry \begin{tabular}{l|l} 
DOI: 10.1159/000495962 & (c) 2018 The Author(s). Published by S. Karger AG, Basel
\end{tabular}

8 Cheng P, Wang J, Waghmare I, Sartini S, Coviello V, Zhang Z, Kim SH, Mohyeldin A, Pavlyukov MS, Minata M, Valentim CL, Chhipa RR, Bhat KP, Dasgupta B, La Motta C, Kango-Singh M, Nakano I: FOXD1-ALDH1A3 Signaling Is a Determinant for the Self-Renewal and Tumorigenicity of Mesenchymal Glioma Stem Cells. Cancer Res 2016;76:7219-7230.

9 van der Heul-Nieuwenhuijsen L, Dits NF, Jenster G: Gene expression of forkhead transcription factors in the normal and diseased human prostate. BJU Int 2009;103:1574-1580.

10 Zhao YF, Zhao JY, Yue H, Hu KS, Shen H, Guo ZG, Su XJ: FOXD1 promotes breast cancer proliferation and chemotherapeutic drug resistance by targeting p27. Biochem Biophys Res Commun 2015;456:232-237.

11 Nakayama S, Soejima K, Yasuda H, Yoda S, Satomi R, Ikemura S, Terai H, Sato T, Yamaguchi N, Hamamoto J, Arai D, Ishioka K, Ohgino K, Naoki K, Betsuyaku T: FOXD1 expression is associated with poor prognosis in non-small cell lung cancer. Anticancer Res 2015;35:261-268.

$\checkmark 12$ Edge SB, Compton CC: The American Joint Committee on Cancer: the 7th edition of the AJCC cancer staging manual and the future of TNM. Ann Surg Oncol 2010;17:1471-1474.

13 Hanahan D, Weinberg RA: Hallmarks of cancer: the next generation. Cell 2011;144:646-674.

14 Klein CA: Parallel progression of primary tumours and metastases. Nat Rev Cancer 2009;9:302-312.

15 Smith SC, Theodorescu D: Learning therapeutic lessons from metastasis suppressor proteins. Nat Rev Cancer 2009;9:253-264.

16 Massague J: TGFbeta signalling in context. Nat Rev Mol Cell Biol 2012;13:616-630.

17 Zhang J, Tian XJ, Zhang H, Teng Y, Li R, Bai F, Elankumaran S, Xing J: TGF-beta-induced epithelial-tomesenchymal transition proceeds through stepwise activation of multiple feedback loops. Sci Signal 2014;7:ra91.

18 Cheng F, Shen Y, Mohanasundaram P, Lindstrom M, Ivaska J, Ny T, Eriksson JE: Vimentin coordinates fibroblast proliferation and keratinocyte differentiation in wound healing via TGF-beta-Slug signaling. Proc Natl Acad Sci U S A 2016;113:E4320-4327.

19 Wu Y, Zhang X, Salmon M, Lin X, Zehner ZE: TGFbeta1 regulation of vimentin gene expression during differentiation of the C2C12 skeletal myogenic cell line requires Smads, AP-1 and Sp1 family members. Biochim Biophys Acta 2007;1773:427-439.

20 Myatt SS, Lam EW: The emerging roles of forkhead box (Fox) proteins in cancer. Nat Rev Cancer 2007;7:847-859.

21 Gao YF, Zhu T, Mao XY, Mao CX, Li L, Yin JY, Zhou HH, Liu ZQ: Silencing of Forkhead box D1 inhibits proliferation and migration in glioma cells. Oncol Rep 2017;37:1196-1202.

22 Dauphin M, Barbe C, Lemaire S, Nawrocki-Raby B, Lagonotte E, Delepine G, Birembaut P, Gilles C, Polette M: Vimentin expression predicts the occurrence of metastases in non small cell lung carcinomas. Lung Cancer 2013;81:117-122.

23 Havel LS, Kline ER, Salgueiro AM, Marcus AI: Vimentin regulates lung cancer cell adhesion through a VAV2Rac1 pathway to control focal adhesion kinase activity. Oncogene 2015;34:1979-1990.

24 Soltermann A, Tischler V, Arbogast S, Braun J, Probst-Hensch N, Weder W, Moch H, Kristiansen G: Prognostic significance of epithelial-mesenchymal and mesenchymal-epithelial transition protein expression in non-small cell lung cancer. Clin Cancer Res 2008;14:7430-7437.

25 Gugnoni M, Sancisi V, Manzotti G, Gandolfi G, Ciarrocchi A: Autophagy and epithelial-mesenchymal transition: an intricate interplay in cancer. Cell Death Dis 2016;7:e2520.

-26 Sun L, Fang J: Epigenetic regulation of epithelial-mesenchymal transition. Cell Mol Life Sci 2016;73:44934515.

27 Ye Z, Zhang X, Luo Y, Li S, Huang L, Li Z, Li P, Chen G: Prognostic Values of Vimentin Expression and Its Clinicopathological Significance in Non-Small Cell Lung Cancer: A Meta-Analysis of Observational Studies with 4118 Cases. PLoS One 2016;11:e0163162. 